\title{
A REVIEW ON VARIOUS FORMULATION OF NANOEMULSIONS IN COSMETICS WITH PLANT EXTRACTS AS THE ACTIVE INGREDIENTS
}

\section{KARINA 0. WISEVA ${ }^{1}$, FRIDA WIDYASTUTI ${ }^{1}$, HISA FAADHILAH ${ }^{1}$, NASRUL WATHONI ${ }^{*}$}

${ }^{1}$ Bachelor Program in Pharmacy, Faculty of Pharmacy, Universitas Padjadjaran, Sumedang 45363, Indonesia, ${ }^{2}$ Department of Pharmacy and Pharmaceutical Technology, Faculty of Pharmacy, Universitas Padjadjaran, Sumedang 45363, Indonesia

Email: nasrul@unpad.ac.id

Received: 03 Aug 2021, Revised and Accepted: 14 Oct 2021

\section{ABSTRACT}

This review conducted to compare the difference of the formulation of nanoemulsion cosmetics by plant extracts between Centella asiatica, seed oil from Rubus idaeus, Phyllanthus urinaria, Garcinia mangostana L., Vellozia sauamata, and Cordyceps militaris extract. Materials and methods was taken from several literature in valid databases that focuses on current status of the formulation of nanoemulsion and its characteristics, nanoemulsion cosmetics, and formulation of nanoemulsion cosmetics that are made by plant extract as the active ingredient. Comparison of the formulas above can be seen from various aspects. Referring to each article, 4 out of 6 formulas use high energy techniques and the rest use low energy techniques. Formula 1, 3, 6 use high energy technique with high pressure homogenization, and formula 4 uses ultrasonication, which is still a high energy technique. Formulas 2 and 5 use low energy techniques, namely formula 2 using Phase Inversion Composition (PIC) and formula 5 using Phase Inversion Temperature (PIT). HighPressure Homogenizer (HPH) is the most widely used for the manufacture of nanoemulsions. In terms of formula, the use of components such as surfactant, cosurfactant, oil phase, and water phase is also different from the 6 formulas presented. Even though all of the six formulas are different, the active ingredients in the form of nanoemulsions need to be tested until finally the goal of the cosmetic product is achieved.

Keywords: Nanoemulsion, Cosmetics, Plants, Extracts

(C) 2021 TheAuthors. Published by Innovare Academic Sciences Pvt Ltd. This is an open access article under theCCBYlicense (https://creativecommons.org/licenses/by/4.0/) DOI: https://dx.doi.org/10.22159/ijap.2021.v13s4.43814 Journal homepage: https://innovareacademics.in/journals/index.php/ijap

\section{INTRODUCTION}

In today's era, the use of nanotechnology is one of the important tools in drug manufacturers, especially nanoemulsions which are used in drug delivery systems and for drug that are difficult to dissolve in water [1]. Nanoemulsion is a carrier system that has convincing potential in solving the problem of delivery of drugs, pesticides or other biological substances and has been widely studied and used. The emulsion itself consists of two or more immiscible liquids in which small droplets of the liquid are dispersed in a continuous phase and have interface boundaries and are a colloid system. From several sources it is said that the droplet size is around 100-500 $\mathrm{nm}$ [2]. The nanoemulsion spheres are homogeneous, isotropic, and thermodynamically unstable colloid systems of two immiscible liquids where one of the liquids is dispersed against the other liquid with nano-scale droplets [3]. The carrier is in the form of solid spheres where its surface is lipophilic and amorphous with a negative charge [4]. This emulsion system has a wide and stable interfacial area, and can also protect the product from adverse environmental conditions [5]. It is very rare to find nanoemulsions containing non-synthetic surfactants, most of them use synthetic surfactants [6].

The method used in the preparation of this nanoemulsion is the direct emulsification method or the titration method and its preparation is quite simple by simply mixing water, oil, cosurfactant, and surfactant according to their respective functions, using mild agitation. Because nanoemulsions are formed directly/ spontaneously, it is recommended not to be too critical in the mixing process. Although this nano-emulsification is spontaneous, to achieve the required balance of this system and the diving process is also small. There are several methods that can be used in the emulsification process, namely using high energy emulsification methods including ultrasonication, high-pressure homogenizers, and microfluidizer or low energy emulsification [7].

There are 3 types of nanoemulsions based on their composition:

1. Oil-in-water nanoemulsion in which oil droplets are dispersed in a continuous phase of water

2. Water-in-oil nanoemulsion in which water droplets are dispersed in an oil continuous phase
3. Bicontinuous nanoemulsion is a nanoemulsion system of water and oil interdissociated in the system

Currently, in pharmaceutical nanoemulsion, nanoemulsion type $\mathrm{W} / \mathrm{O}$ is more popular than $\mathrm{W} / \mathrm{O}$ because $0 / \mathrm{W}$ nanoemulsion is easier to wash, compatible with water, safer and more widely used in drug delivery systems [8].

The following is the structure of a water-in-oil and oil-in-water emulsion:

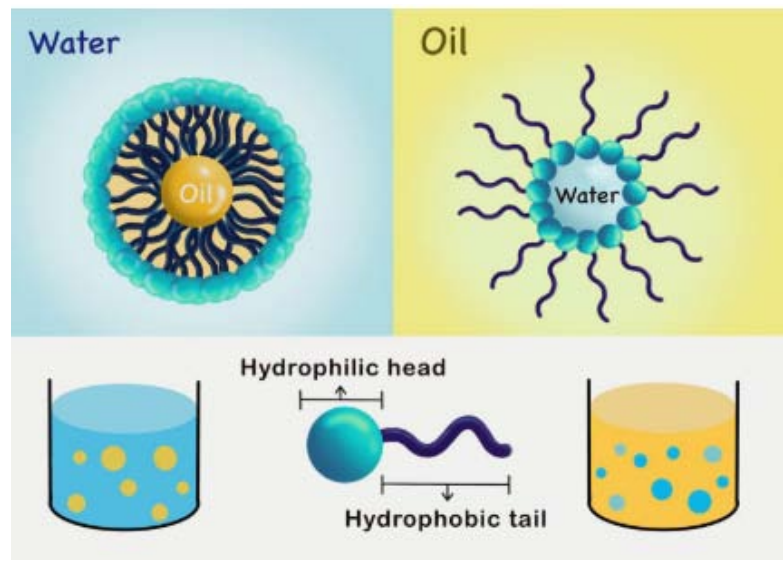

Note: Adapted from Ashaolu, 2021.

Fig. 1: Structure of Emulsion $0 / W$ and $W / 0$. Nanoemulsion is a system that is immiscible that consists of water and oil, and can be performed in a single phase by surfactants and cosurfactants or other emulsifiers. This system also has high thermodynamics and stability [9]

The stability of the interfaces of the three types depends on the correct combination of surfactants or co-surfactants. The main difference between emulsion and nanoemulsion is its formed, exhibit good 
kinetic stability, but are thermodynamically unstable and can readily form separate phases. Another important thing that makes the difference is in terms of appearance, where the emulsion is a bit cloudy while the nanoemulsion is clear. The emulsion requires a lot of energy in its manufacture while the nanoemulsion does not [4].

This emulsion system has been widely applied in various industrial sectors, such as the food, cosmetics, and pharmaceutical industries. In the cosmetic field, nanoemulsions are used as lotions, creams and moisturizers. In this delivery system, the active constituents can be easily absorbed so that they can provide the effective action based on its characteristic properties with a small size of the droplet and ability to reduce the water loss from the skin. In this nanoemulsion technique, cosmetic formulations are expected to produce rapid penetration of the active ingredients against the skin due to the large surface area of the droplets. Even skin with a rough surface, it is proven that using nanoemulsion can still be penetrated properly. In this nanoemulsion formulation only requires a smaller amount of surfactant. For example, the surfactant needed for microemulsion is around $20-25 \%$ but only $5-10 \%$ of the surfactant is sufficient for nanoemulsion [10].

At this time, there has been a lot of research and interest in cosmetic formulations containing antioxidant active ingredients from natural ingredients. Polyphenols and flavonoids are compounds that are being studied for their antioxidant properties in plant extracts. Nanoformulation techniques containing plant extracts are an alternative for topical cosmetics [11]. Examples of plant extracts that can be used as cosmetics with nanoformulations include Centella asiatica extract, seed oil from Rubus idaeus, and Phyllanthus urinaria extract.

The advantages and disadvantages of nanoemulsion cosmeticsNanoemulsions consist of very good oil-in-water dispersion and also the diameter of the droplets is small, less than $100 \mathrm{~nm}$. When compared to others, for example microemulsions, it is metastable and naturally very brittle. Nanoemulsions can quickly penetrate the skin because they have good sensory properties with good penetration, and biophysical properties that have good hydration capacity. Many products have been produced from this nanoemulsion technique, such as clear gels such as crystals with different rheological properties, transparent milks, lotions, and water-like fluids. In addition, its manufacture requires only a small amount of energy [10]. Cosmetic products made with this nanoemulsion do not cause flocculants, creaming agents, coalescence, and no sediment, so it can be said to be better than other emulsion techniques in the manufacture of cosmetics [9]. In addition, in terms of cosmetic appearance, this nanoemulsion looks more glossy on the skin and the infiltration has also increased [12].

This nanoemulsion system has been widely applied due to its properties, purity, simplicity and ability to increase drug bioavailability and sterilization through filtration of the dissolved drug. But besides the advantages possessed by nanoemulsions, there are also many sources who say that nanoemulsions with $r<100 \mathrm{~nm}$ are thermodynamically unstable and cause damage to the system with respect to various instability mechanisms in it. The small size of the nanoemulsion can also cause irreversible instability as a result of the Ostwald ripening or coalescence mechanism. When small droplets are dissolved in larger particles, it can affect the change in the radius of the drop to decrease [10].

This review conducted to compare the difference of the formulation of nanoemulsion cosmetics by plant extracts between Centella asiatica, seed oil from Rubus idaeus, Phyllanthus urinaria, Garcinia mangostana L., Vellozia sauamata, and Cordyceps militaris extract. From the six formulas, we will see the different aspects, and which one is the best formula that is suitable and worthy to become natural nanoemulsion cosmetics to be used by people as cosmetics.

\section{MATERIALS AND METHODS}

This review is based on the literature obtained from Scopus, Pubmed and Google Scholar using the keywords "nanoemulsion", "nanoemulsion cosmetics, and "nanoemulsion cosmetics from plant extracts". The database is limited to obtain the specific topic in comparison between formulation of nanoemulsion cosmetic with
Centella asiatica, seed oil from Rubus idaeus, Phyllanthus urinaria, Phyllanthus urinaria, Garcinia mangostana L., Vellozia sauamata, and Cordyceps militaris extract. The literature that we used in this review is written from 2011-2020.

\section{RESULTS}

\section{Manufacturing of cosmetic nanoemulsion}

\section{a. Formulation}

Cosmetics can be seen by their properties based on their ability to provide active ingredients and also based on their textural properties. This nanoemulsion in topical form on the skin exhibits higher performance and bioavailability because it provides a uniform formation of a thin lipid film [13]. Formulating this cosmetic has many important variables, for example the choice of emollient excipient needs attention in its physicochemical properties because it will affect dispersion, final sensory properties, and oleosity [14]. There are some elements that need to be considered in the manufacture of nanoemulsions, including the type of components, the amount of components in the system, the emulsification process, and the conditions [15]. The main components in nanoemulsions are oils, surfactants, cosurfactants, active ingredients, and the water phase (can be preservatives, viscosity, $\mathrm{pH}$, and penetration enhancing agents, buffer salts) [16]. However, it is also mentioned in another article that the components of nanoemulsion are oil, water, and surfactants [17].

\section{Oils}

Functions in dissolving hydrophobic materials and as an [16]. Oil phases that can be used in nanoemulsions, for example, are essential oils, free fatty acids, and others with non-polar molecular properties. The physical and chemical properties (viscosity, water solubility, density, polarity, refractive index, and interfacial tension) of the oil phase have an influence on the stability, formation, and characteristics of nanoemulsions [18].

\section{Surfactants}

Function in stabilizing nanoemulsions and reducing droplet size. These surfactants can be used singly or in combination. Non-ionic surfactants are preferred in cosmetic manufacturing because of their effective reduction of interface stress and avoidance of skin irritation [16]. The addition of surfactants will reduce the interfacial tension. When the oil phase and water phase are mixed, a temporary emulsion will be formed and due to coalescence of the dispersed granules, the mixture will separate into two phases [18]. Mixed surfactants can also be used to improve stability in nanoemulsions due to the broad-chain length distribution [19].

\section{Cosurfactants}

Used in classic emulsions and cosmetic products. One type of surfactant is polyols. As for examples, namely Glycerin, propylene glycol, PEG300, PEG400, poloxamer [16].

\section{Water phase}

Important in nanoemulsion stability [16]. In the aqueous phase, water can be mixed with other polar molecules. The aqueous phase also determines the formulation, stability and physicochemical quality of the nanoemulsion which is determined by $\mathrm{pH}$, ionic strength, polarity, density, rheology, refractive index, interfacial tension, and phase behavior of the aqueous phase, which depends on the concentration of the components used. and their types [18].

\section{Active ingredients}

The active ingredients used for cosmetics aim to change the physiology of skin cells that make them healthier [20] and also improve or protect the appearance of the human body [21] Nanoemulsions are intended in cosmetics for optimal delivery of active ingredients to the skin [22]. Herbs that can be used are those that have active ingredients with the specific purpose and function of the cosmetic itself. Herbal active ingredients derived from these plants need standardization of raw materials, free from contaminants (microbes, insecticides, heavy metals, etc.), no abnormalities in color, odor, and signs of decomposition [23]. 


\section{b. Method}

The method that can be used in the manufacture of cosmetic nanoemulsions is the high-energy and low-energy mechanical dispersion methods [16]. The method using high energy has an emulsification method including ultrasonication, high-pressure homogenizer (HPH), microfluidization and membrane emulsification. The low energy method has emulsification methods including phase inversion temperature (PIT), phase inversion composition (PIC) and solvent diffusion [15].

\section{Methods of preparation}

\section{High-energy}

The manufacture of nanoemulsions with this high energy technique uses devices to supply energy to increase the water/oil interface which then produces submicron droplets (mechanical energy breaks the droplets into smaller ones) [24].

a) High pressure homogenization or also known as HPH is a manufacturing method to produce nanoemulsions with submicron size by combining hydraulic shear, cavitation, and turbulence. This size is controlled by the application of pressure and the number of homogenization cycles of each stage. The smaller size is also affected by the homogenization temperature [16]. HPH is the most widely used for the manufacture of nanoemulsions. The result of the particle size of the nanoemulsion is very low, reaching $1 \mathrm{~nm}$ [25].

b) Microfluidization uses a microfluidizer device in which the coarse emulsion will be forced through a small channel with a certain configuration under the influence of high pressure injection until the impingement area is then produced fine particles [16].

c) Ultrasonication has the principle of the cavitation method. To destroy coarse emulsions using a probe that emits ultrasonic waves. Time variation, energy input, oil and surfactant concentration ratio control, can control droplet size [16].

\section{Low-energy}

The manufacture of nanoemulsions with this low energy technique without using any device or energy, the manufacture is through spontaneous emulsification. This technique relies on the chemical energy present in the system components [24].

a) Phase inversion temperature or also known as PIT has the principle of cooling method. PIT is carried out by mixing together oil water, and surfactants at room temperature and an $0 / \mathrm{W}$ emulsion is produced. Heat is carried out gradually then this nonionic surfactant becomes lipophilic and mixed in the oil phase [16].

b) Phase inversion composition or also known as PIC has the principle of dilution method. PIC is carried out by adding water and continuously stirring in a mixture of surfactants and oil. PIC has limitations that require titration [16].

Spontaneous emulsification has the principle of dispersion and condensation method. It was carried out by diluting the $\mathrm{o} / \mathrm{w}$ microemulsion with water, which then the cosurfactant diffused along the water continuous phase until it reached a nanoemulsion (which is thermodynamically unstable). After that, the water-soluble compound is rapidly diffused from the dispersed phase to the continuous phase [16].
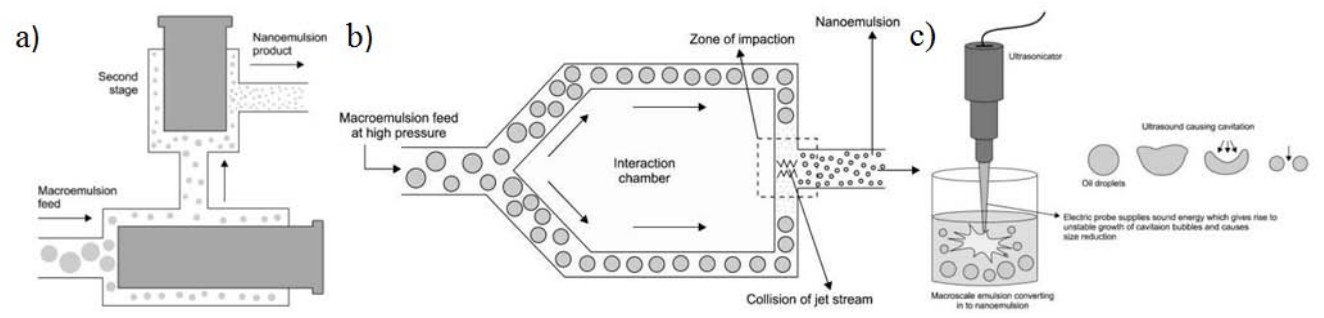

Note. Adapted from Kumar et al., 2019.

Fig. 2: High energy methods: a) high-pressure homogenization; b) microfluidization; c) ultrasonication [5]
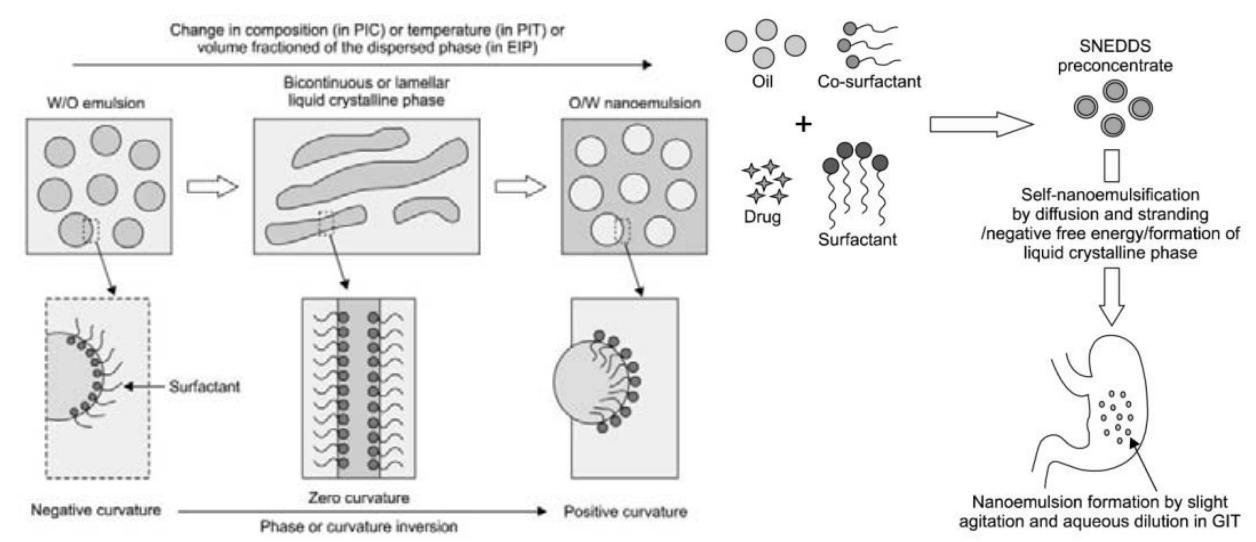

Note. Adapted from Kumar et al., 2019.

Fig. 3: Low energy methods: a) phase inversion techniques; b) self emulsifications techniques [5]

\section{Comparison of nanoemulsion cosmetic formulations}

a. Formulation 1 (Lotion containing Centella asiatica Extract as anti-photoaging)

The ingredients for this formula are ethanolic extract of Centella asiatica as the active ingredient, olive oil, stearic acid, cetyl alcohol, tween-80 (polysorbate) as the surfactant, $96 \%$ ethanol and propylene glycol as the co-surfactant, and for the water phase there are aquademineralisata, propylene glycol, tetraethylammonium (TEA), propylparaben, methyl paraben, and isopropyl myristate [26].

b. Formulation 2 (Red raspberry (Rubus idaeus) seed oil as antioxidant) 
The ingredients for this formula are red raspberry seed oils as the active ingredient, isostearyl isostearate and tocopheryl acetate as the oils, polyoxyethylene-20 sorbitan monooleate (polysorbate 80 ) as the surfactant, and for the water phase are glycerol, hydroglycolic extract of red raspberry fruit (water, propylene glycol, Rubus idaeus fruit extract), and hydroglycolic extract of french oak fruit (glycerin, Quercus petraea fruit extract) [27].

\section{c. Formulation 3 (Phyllanthus urinaria for skin anti aging)}

The ingredients for this formula are powder (ethanolic dried extract) of Phyllanthus urinaria as the active ingredient, PKOes (palm kernel oil esters), cetyl alcohol 99\%, glyceryl monostearate, tween 80 (polysorbate) and span 80 as the surfactants, buffer phosphate and sodium benzoate as the water phase [28].

d. Formulation 4 (Mangostin extract-loaded virgin coconut oil as antioxidants and antimicrobial agent)
The ingredients for this formula are mangostin (Garcinia mangostana L.) extract as the active ingredient, Virgin Coconut Oil (VCO),Span 20 and tween 20 as surfactants, propylen glycol and aquos continuous phase as the water phase [29].

e. Formulation 5 (Hydroalcoholic extracts of Vellozia squamata as antioxidants)

The ingredients for this formula are hydroalcoholic extract of Vellozia squamata as the active ingredient, babacu oil, castor oil, PEG-40 hydrogenated and sorbitan monostearate as the surfactants, and distilled water [30].

\section{f. Formulation 6 (Cordyceps militaris extracts as antioxidants)}

The ingredients for this formula are Cordyceps militaris extracts as the active ingredients, sugar squalane, jojoba and argan oil, tween 20 , tween 80, and tween 85 as the surfactants, and deionized water [31].

Table 1: Various methods used for preparing nanoemulsions containing plant extract

\begin{tabular}{|c|c|c|c|}
\hline Formula & Plant & Method & References \\
\hline 1 & Centella asiatica & High-pressure Homogenizer (HPH) & {$[14]$} \\
\hline 2 & Rubus idaeus & Phase Inversion Composition (PIC) & [15] \\
\hline 3 & Phyllanthus urinaria & High-pressure Homogenizer (HPH) & [16] \\
\hline 4 & Garcinia mangostana L. & Ultrasonication & [17] \\
\hline 5 & Vellozia squamata & Phase Inversion Temperature (PIT) & [18] \\
\hline 6 & Cordyceps militaris & High-pressure Homogenizer (HPH) & [19] \\
\hline
\end{tabular}

\section{DISCUSSION}

\section{Comparison of formulations}

Comparison of the formulas above can be seen from various aspects. Referring to each article, 4 out of 6 formulas use high energy techniques and the rest use low energy techniques. Formula 1, 3, 6 use high energy technique with high pressure homogenization, and formula 4 uses ultrasonication, which is still a high energy technique. Formulas 2 and 5 use low energy techniques, namely formula 2 using phase inversion composition (PIC) and formula 5 using phase inversion temperature (PIT) $[26-30,32]$. HPH is the most widely used for the manufacture of nanoemulsions [25]. Although generally using $\mathrm{HPH}$, the method of making nanoemulsions can also be adapted to the formula made, for example formula 5 using PIT shows which stable nanoemulsion is appropriate for this extract for topical use [30].

In formula 1, several evaluations of nanoemulsions were carried out, including physical appearance, $\mathrm{pH}$, particle size, transmission electron microscopy, rheology, physical appearance, and skin penetration [26]. Formula 2 is evaluated by Raman spectroscopy, microscopic analysis, $\mathrm{pH}$, electrical conductivity, rheology and texture, in vitro antioxidants, storage tests, in vitro tests of biological activity [27]. Formula 3 evaluates physical characters ( $\mathrm{pH}$, particle size, zeta potential, TEM), rheological tests, and in vitro tests for formula tests [28]. Formula 4 evaluates including determining $\mathrm{pH}$, average particle diameter, zeta potential, morphology, nanoemulsion, antioxidant activity [29]. Formula 5 by determining the size of nanoparticles, $\mathrm{pH}$, process, process of rifugation, thermal stress, and rheology [30]. Formula 6 evaluates including physical characterization (organoleptic), size, PDI, zeta potential, and of nanoemulsion [31].

The evaluation of nanoemulsions as mentioned above aims to assess the nanoemulsions. As with the determination of $\mathrm{pH}$, the $\mathrm{pH}$ range for the skin is 4-6, depending on the individual and the skin area, this $\mathrm{pH}$ determines irritation of the skin. Another example for particle size, the small particle size in nanoemulsions functions in uniform deposition and increases penetration in the skin [28].

In terms of formula, the use of components such as surfactant, cosurfactant, oil phase, and water phase is also different from the 6 formulas presented. However, in the selection of surfactants, all formulas use non-ionic surfactants where this type of surfactant compared to anionic or cationic surfactants shows relatively nontoxic and non-irritating properties. Use in combination with cosurfactants is also intended to reduce the interface of the oil and water layer [26]. The choice of surfactant is also an important factor in the formulation because of its use in reducing surface and droplet properties [28]. Thus, the composition of the formula also requires further study in order to obtain the maximum formula.

The active ingredients of the 6 formulas above have different functions but the goal is the same in the cosmetic field. An example of the above formula, antioxidants are one strategy to protect the skin and quench free radicals. The use of antioxidants in this cosmetic formulation needs to meet various conditions, including being stable with physicochemical properties or with other additives, reaching the stratum corneum, and reaching deeper layers of the skin without entering the systemic circulation [32]. Even though they are different, the active ingredients in the form of nanoemulsions need to be tested until finally the goal of the cosmetic product is achieved.

\section{Characterics of nanoemulsion}

Nanoemulsion itself has the characteristics of good stability, controlled release, and high ability to increase drug bioavailability. In addition, nanoemulsions have the ability to be manufactured with high flexibility so that the obtained portion of the drug is different with different characteristics [33]. Nanoemulsion was produced and characterized by $\mathrm{pH}$, zeta potential, viscosity, droplet size and PDI. $\mathrm{pH}$ of the formulations was measured in the potentiometer model 922 METER [34]

Zeta potential-Zeta potential is included in the characteristics of cosmetic nanoemulsions due to its composition and stable value for the formulation. The count rate is maintained between 100 and 300 kcps. Particles with a zeta potential value more positive than $+30 \mathrm{mV}$ or more negative than $30 \mathrm{mV}$ are considered stable [35]. Zeta potential is a parameter that describes the function of surface charge on droplets and is usually measured using a zetasizer. Sample from other formulations placed in zeta cuvette and reading in $\mathrm{mV}$ [36].

Droplet size and PDI-The droplet size and the PDI (Polydispersity Index) of the Nanoemulsion were determined using Zetasizer S90 [34]. The mean droplet size diameter and index were determined by photon correlation spectroscopy. The analyzes were made in an angle of $90^{\prime \prime}$ (triple) at room temperature (25 $5 \mathrm{C}$ ). Then the $\mathrm{pH}$ value was determined at room temperature (25-5 C) [37]. Low PDI shows that formulations have to produce a narrow size distribution and lower PDI support a much lower size distribution [38]. 
Viscosity-Viscosity is a parameter crucial to exemplify and gauge stability liquid and semi-solid preparations, on top of the efficient release of active ingredients from the carrier in formulations. In fact, viscosity is very much dependent on the compositions of surfactant, water and oil components of the emulsion along with their concentrations [39]. Also, parameters viz. viscosity, conductivity and dielectric methods offer critical insights into the macroscopic level of the formulated emulsion [40]. It is worth mentioning here that viscosity determination becomes important when the percentage of the oil component is increased. This is because viscosity of an emulsion increases correspondingly with further addition of oil, which may affect the sensory attribute of the resultant formulation [41].

Some of the critical characteristics shown by cosmetic formulations based on nanotechnology are: active cosmetic ingredient, shape, size, additional features and charge [42]. Cosmetic ingredients have been used in the cosmetic industry for more than $30 \mathrm{y}$. Nano-derived cosmetic materials are insoluble or bio-persistent materials and are intentionally made with one or more external dimensions or internal structures on a scale from 1 to $100 \mathrm{~nm}$ [43]. Nanotechnology produces materials of various types at the nanoscale level. Nanoparticles are a broad class of size materials that if one dimension is less than $100 \mathrm{~nm}$ depending on the overall shape, these materials can be 0D, 1D, 2D or 3D [44]. The use of nanoparticles in cosmetics has shown many benefits of nano-particles, such as $\mathrm{Ag}, \mathrm{Au}$, and Pt. Particle size distribution is one of the most important physical characteristics, because to get the right formulation and get the most important variables from the manufacture of nano cosmetics. The optimal value of nanocosmetics is that which has the lowest particle size and high stability formulation [45].

Nanoparticles of different morphology and chemical composition are commonly incorporated in cosmetic formulations to take advantage of shape and size related activities that can intensify product performance and are responsible for toxicology effects. In contrast to UV absorbers, several types of inorganic oxide-based nanoparticles, such as $\mathrm{ZnO}$-nanoparticles, TiO2-nanoparticles, $\mathrm{ZrO2}$ nanoparticles, or $\mathrm{CeO} 2$ nanoparticles, are added to sunscreen preparations as physical UV filters because of their ability to scatter and reflect UV radiation. This nanostructured material has the ability to attenuate UV radiation and results in an increased capacity to withstand light UV. In one study, calcium-doped $\mathrm{CeO} 2$ has shown better filtering of shorter UV-A radiation. Cerium phosphate and cerium-titanium pyrophosphates have also been shown to be effective substitutes for $\mathrm{ZnO}$ and $\mathrm{TiO} 2$ nanoparticles in sunscreen formulations. Nanoparticles such as clay and silica are silica added in cosmetic formulations as thickeners. In addition, nanoscale inorganic-organic hybrid materials are also used in cosmetic products as thickening agents. For example, hydrophobicty grafted perfluorocarbon nanoparticles of hydrophobically modified $\mathrm{SiO} 2$ allow the development of nano-sized cross-linked polymer network structures, which protect the skin from organophosphate exposure and toxicity [12].

\section{Evaluation of nanoemulsion cosmetic formulations}

There are several studies and tests that must be carried out to know whether the formula or product qualify or not:

\section{a. Biological study}

To get good results, which can penetrate the pores and the carrier material interacts with the stratum corneum layer, an evaluation is carried out first using the human skin organotypic culture (HSOC) model which is also used to test toxicity [46].

\section{b. Stability study}

The preliminary stability of the nanoemulsion preparation was evaluated at $24 \mathrm{~h}$ by centrifuge and thermal stress analyses. Stability was assessed by macroscopic emulsion observation and droplet size analysis. The purpose of these tests was to select a stable, lowsurfactant formulation with a nanoemulsion-size droplet and stable physicochemical properties. The selected nanoemulsion was prepared in triplicate, and the samples were stored at $25 \pm 2{ }^{\circ} \mathrm{C}, 40 \pm 2$ ${ }^{\circ} \mathrm{C}$ and $5 \pm 2{ }^{\circ} \mathrm{C}$. Tests were performed at $24 \mathrm{~h}, 7,15,30,60$ and $90 \mathrm{~d}$ after preparation. The analysis measurements were droplet size, $\mathrm{pH}$ value and electrical conductivity [47].

\section{c. Organoleptic study}

In the organoleptic test, observations were made in the form of physical, color, and odor to the results of the formulation that had been made. The homogeneity of the preparation is also seen if you want to make lotion, or preparations that absorb the skin. The trick is that the preparation that has been made is smeared on glass pieces and then seen for homogeneity, if there are no coarse grains it can be said to be homogeneous [48].

\section{d. Calculating yield percentage}

From the formula that has been made, it is seen how efficiently the nanoparticles are calculated by comparing the total amount of drug and polymer in the formula with the yield percentage formula [49].

\section{e. pH test and skin hydration}

The $\mathrm{pH}$ test is carried out by observing the acidity level of the preparation to ensure that the resulting formulation does not cause irritation to the skin, and can be measured using a $\mathrm{pH}$ meter. The evaluation of $\mathrm{pH}$ characteristic can be done by direct immersion of the electrode in samples of the formulation [34]. Seeing the level of hydration in the skin also needs to be done, it has been proven to cause hydration to the skin if it shows a value of 0-150 AU (arbitrary units). Hydration is one of the important parameters which see changes in the permeability of the skin. Skin permeability is strongly influenced by the composition of the emulsion, the presence or absence of charge on the granules and surfactants [50].

\section{f. In vitro study}

The in vitro method used can use embryonic chickens that can be purchased from hatcheries. The chicken was waited for $10 \mathrm{~d}$ after fertilization and kept at a temperature of $37{ }^{\circ} \mathrm{C} \pm 2$. Then the egg's upper shell was made into small incisions using scissors. Next, the white membrane on the inside of the egg is pulled out to make the chorioallantoic membrane transparent with blood vessels. The sample formulation was weighed as much as $0.3 \mathrm{~g}$ and then applied to the egg membrane. Count 20 seconds and then wash with $5 \mathrm{ml}$ of salt solution. See whether there is irritation that appears. Sodium Lauryl Sulfate (SLS) was used as a positive control, salt solution $(0.9 \%)$ and purified water as a negative control. Then the resulting irritation is evaluated according to the Luepke scale and classified [22].

\section{g. In vivo study}

This method can use several rats as negative control, positive formulation and formulation. Then it is injected into the left ear for normal, and the test is injected in the right ear. For positive control, the dose of dexamethasone can be adjusted according to the dose. Then after being injected all four hours later, the mice were sacrificed and their thickness measured $(\mathrm{mm})$ and considered to assess the edema mass (mg). In both methods, the thickness values for the right ear were discounted from the right ear against all groups and the comparison to control was negative [51].

Nowadays, nanotechnology is one of the developed innovations that can be used as a drug delivery system, one of the applications in nanotechnology is nanoemulsion. There are so many benefits and advantages that we can take from nanoemulsion cosmetics, especially by its characteristic, it can cover some of the disadvantages in other drug delivery systems. Rapid penetration is one of the best result that can we see in this application. So it can be said that this system is better than other emulsion techniques in the manufacture of cosmetics, specifically in topical cosmetics. Pharmaceutical field and industry scale can provide their products by this technology as a recency.

\section{CONCLUSION}

Nanoemulsion is a good technique to be used in the pharmaceutical world, one of which is in the manufacture of cosmetics. It's easy and fast penetration into the skin makes this system suitable for application in cosmetics and skincare such as lotions, creams, moisturizers. The active substances can also be obtained from various plant extracts that have a repairing effect on the skin. In the discussion of this review, it is seen that the three formulas have different forms and compositions depending on the method used. In 
formula 1, the formulation uses Centella asiatica extract, formula 3 Phyllanthus urinaria, and also formula 6 Cordyceps militaris are use the high-pressure homogenization (HPH) method where in this method a submicron size is produced by combining hydraulic shear, cavitation, and turbulence. For formula 2 with Rubus idaeus extract, using the method of phase inversion composition (PIC) which in this method uses the principle of adding water and continuously stirring in a mixture of surfactants and oil. Formula 4 Garcinia mangostana $\mathrm{L}$. uses ultrasonication that has the principle of the cavitation method. The last one, formula 5 Vellozia squamata using phase inversion temperature (PIT) that has the principle of cooling method.

\section{FUNDING}

Nil

\section{AUTHORS CONTRIBUTIONS}

All authors have contributed equally.

\section{CONFLICT OF INTERESTS}

Declared none

\section{REFERENCES}

1. Alam MS, Ali MS, Alam N, Siddiqui MR, Shamim M, Safhi MM. In vivo study of clobetasol propionate loaded nanoemulsion for topical application in psoriasis and atopic dermatitis. Drug Invent Today. 2013;5(1):8-12. doi: 10.1016/j.dit.2013.02.001.

2. AN, Kovooru L, Behera AK, Kumar KPP, Srivastava P. A critical review of synthesis procedures, applications and future potential of nanoemulsions. Adv Colloid Interface Sci. 2021;287:102318. doi: 10.1016/j.cis.2020.102318, PMID 33242713.

3. Patel RB, Thakore SD, Patel MR. Recent survey on patents of nanoemulsions. Curr Drug Deliv. 2016;13(6):857-81. doi: $10.2174 / 1567201812666150901111930$, PMID 26324230.

4. Sharma N. Preparation and optimization of nanoemulsions for targeting drug delivery INM-318 view project development of formulation for universal decontamination of CBRN agents with minimal waste generation view project. Int J Drug Dev Res. 2013;5:37-48.

5. Kumar M, Bishnoi RS, Shukla AK, Jain CP. Techniques for formulation of nanoemulsion drug delivery system: a review. Prev Nutr Food Sci. 2019;24(3):225-34. doi: 10.3746/pnf.2019.24.3.225, PMID 31608247.

6. Zhou H, Yue Y, Liu G, Li Y, Zhang J, Gong Q, Yan Z, Duan M. Preparation and characterization of a lecithin nanoemulsion as a topical delivery system. Nanoscale Res Lett. 2009;5(1):22430. doi: 10.1007/s11671-009-9469-5, PMID 20652152.

7. Najafi Taher R, Amani A. Nanoemulsions: colloidal topical delivery systems for antiacne agents-a mini-review. Nanomed Res J. 2017;2(1):49-56.

8. Pengon S, Chinatangkul N, Limmatvapirat C, Limmatvapirat S. The effect of surfactant on the physical properties of coconut oil nanoemulsions. Asian J Pharm Sci. 2018;13(5):409-14. doi: 10.1016/j.ajps.2018.02.005, PMID 32104415.

9. Ashaolu TJ. Nanoemulsions for health, food, and cosmetics: a review. Environ Chem Lett. 2021:1-15. doi: 10.1007/s10311. 021-01216-9, PMID 33746662

10. Chellapa P, Ariffin FD, Eid AM, Almahgoubi AA, Mohamed AT, Issa YS. Nanoemulsion for cosmetic application. Available from: www.ejbps.com. [Last accessed on 02 Jul 2021]

11. Quintao FJO, Tavares RSN, Vieira Filho SA, Souza GHB, Santos ODH. Hydroalcoholic extracts of Vellozia squamata: study of its nanoemulsions for pharmaceutical or cosmetic applications. Rev Bras Farmacognosia. 2013;23(1):101-7. doi: 10.1590/S0102-695X2013005000001.

12. Bilal M, Iqbal HMN. New insights on unique features and role of nanostructured materials in cosmetics. Cosmetics. 2020;7(2):1-16.

13. Sonneville Aubrun O, Yukuyama MN, Pizzino A. Application of nanoemulsions in cosmetics. Nanoemulsions: Formulation, Applications and Characterization. Elsevier Inc; 2018.

14. Ribeiro RC, Barreto SMAG, Ostrosky EA, da Rocha-Filho PA Verissimo LM, Ferrari M. Production and characterization of cosmetic nanoemulsions containing Opuntia ficus-indica (L.) Mill extract as moisturizing agent. Molecules.
2015;20(2):2492-509. doi: 10.3390/molecules20022492, PMID 25648593.

15. Yukuyama MN, Ghisleni DDM, Pinto TJA, Bou-Chacra NA Nanoemulsion: process selection and application in cosmetics-a review. Int J Cosmet Sci. 2016;38(1):13-24. doi: 10.1111/ics.12260, PMID 26171789

16. Faria Silva AC, Costa AM, Ascenso A, Ribeiro HM, Marto J, Gonçalves LM. Nanoemulsions for cosmetic products. Nanocosmetics. 2020;1:59-77.

17. Dasgupta N, Ranjan S, Gandhi M. Nanoemulsion ingredients and components. Environ Chem Lett. 2019;17(2):917-28. doi: 10.1007/s10311-018-00849-7.

18. Azrini N, Azmi N, Elgharbawy AAM, Motlagh SR, Samsudin N. Nanoemulsions: factory for food, pharmaceutical and cosmetics. Processes. 2019;7(617):1-34.

19. Lu WC, Huang DW, Wang CR, Yeh CH, Tsai JC, Huang YT, Li PH. Preparation, characterization, and antimicrobial activity of nanoemulsions incorporating citral essential oil. J Food Drug Anal. 2018;26(1):82-9. doi: 10.1016/j.jfda.2016.12.018, PMID 29389592.

20. Pimentel FB, Alves RC, Rodrigues F, Oliveira MBPP. Macroalgae-derived ingredients for cosmetic industry-an update. Cosmetics. 2018;5(1):4-9.

21. Dixit D, Reddy CRK. Non-targeted secondary metabolite profile study for deciphering the cosmeceutical potential of red marine macro alga jania rubens- an LCMS-based approach. Cosmetics. 2017;4(4):1-17. doi: 10.3390/cosmetics4040045.

22. Rocha-Filho PA, Ferrari M, Maruno M, Souza O, Gumiero V. In vitro and in vivo evaluation of nanoemulsion containing vegetable extracts. Cosmetics. 2017;4(3). doi: $10.3390 /$ cosmetics4030032.

23. Algin Yapar E. Herbal cosmetics and novel drug delivery systems. Indian J Pharm Educ Res. 2017;51(3):S152-8.

24. Che Marzuki NH, Wahab RA, Abdul Hamid M. An overview of nanoemulsion: concepts of development and cosmeceutical applications. Biotechnol Biotechnol Equip. 2019;33(1):779-97. doi: $10.1080 / 13102818.2019 .1620124$.

25. Pandey V, Shukla R, Garg A, Kori ML, Rai G. Nanoemulsion in cosmetic: from laboratory to market. Nanocosmetics INC 2020;201:327-47.

26. Hanifah M, Jufri M. Formulation and stability testing of nanoemulsion lotion containing Centella asiatica extract. J Young Pharm. 2018;10(4):404-8. doi: 10.5530/jyp.2018.10.89.

27. Gledovic A, Janosevic Lezaic A, Krstonosic V, Djokovic J, Nikolic I, Bajuk-Bogdanovic D, Antic Stankovic J, Randjelovic D, Savic SM, Filipovic M, Tamburic S, Savic SD. Low-energy nanoemulsions as carriers for red raspberry seed oil: formulation approach based on Raman spectroscopy and textural analysis, physicochemical properties, stability and in vitro antioxidant/ biological activity. PLOS One. 2020;15(4):e0230993. doi: 10.1371/journal.pone.0230993. PMID 32298275.

28. Mahdi ES, Noor AM, Sakeena MH, Abdullah GZ, Abdulkarim MF, Sattar MA. Formulation and in vitro release evaluation of newly synthesized palm kernel oil esters-based nanoemulsion delivery system for $30 \%$ ethanolic dried extract derived from local Phyllanthus urinaria for skin antiaging. Int J Nanomedicine. 2011;6:2499-512. doi: 10.2147/IJN.S22337, PMID 22072884.

29. Sungpud C, Panpipat W, Chaijan M, Sae Yoon AS. Technobiofunctionality of mangostin extract-loaded virgin coconut oil nanoemulsion and nanoemulgel. PLOS One. 2020;15(1):e0227979. doi: 10.1371/journal.pone.0227979, PMID 31995599.

30. Quintao FJO, Tavares RSN, Vieira Filho SA, Souza GHB, Santos $\mathrm{ODH}$. Hydroalcoholic extracts of Vellozia squamata: study of its nanoemulsions for pharmaceutical or cosmetic applications. Rev Bras Farmacognosia. 2013;23(1):101-7. doi: 10.1590/S0102-695X2013005000001.

31. Marsup P, Yeerong K, Neimkhum W, Sirithunyalug J, Anuchapreeda S, To-Anun C, Chaiyana W. Enhancement of chemical stability and dermal delivery of Cordyceps militaris extracts by nanoemulsion. Nanomaterials (Basel). 2020;10(8):1-26. doi: 10.3390/nano10081565, PMID 32784892 . 
32. Van Tran V, Loi Nguyen T, Moon JY, Lee YC. Core-shell materials, lipid particles and nanoemulsions, for delivery of active anti-oxidants in cosmetics applications: challenges and development strategies. Chem Eng J. 2019;368:88-114. doi: 10.1016/j.cej.2019.02.168.

33. Abdel Samie SM, Nasr M. Food to medicine transformation of stilbenoid vesicular and lipid-based nanocarriers: technological advances. Drug Delivery Aspects. Elsevier Inc; 2020. p. 227-45.

34. Zanela da Silva Marques T, Santos Oliveira R, Betzler de Oliveira de Siqueira L, Cardoso VDS, de Freitas ZMF, Barros RCDSA, Villa ALV, Monteiro MSSB, Dos Santos EP, Ricci Junior E. Development and characterization of a nanoemulsion containing propranolol for topical delivery. Int J Nanomed. 2018;13:2827-37. doi: 10.2147/IJN.S164404, PMID 29785109.

35. Mohd Taib SH, Abd Gani SS, Ab Rahman MZ, Basri M, Ismail A, Shamsudin R. Formulation and process optimizations of nanocosmeceuticals containing purified swift let nest. RSC Adv. 2015;5(53):42322-8. doi: 10.1039/C5RA03008K.

36. Ghareeb MM, Neamah AJ. Formulation and characterization of nimodipine nanoemulsion as ampoule for oral route. Int J Pharm Sci Res. 2017;8(2):591-602.

37. Pereira TA, Guerreiro CM, Maruno M, Ferrari M, Rocha-Filho PA. Exotic vegetable oils for cosmetic $0 / \mathrm{W}$ nanoemulsions: in vivo evaluation. Molecules. 2016;21(3):248. doi: 10.3390/molecules21030248, PMID 26927034.

38. He W, Tan Y, Tian Z, Chen L, Hu F, Wu W. Food proteinstabilized nanoemulsions as potential delivery systems for poorly water-soluble drugs: preparation, in vitro characterization, and pharmacokinetics in rats. Int J Nanomed. 2011;6:521-33. doi: 10.2147/IJN.S17282, PMID 21468355.

39. Yue PF, Lu XY, Zhang ZZ, Yuan HL, Zhu WF, Zheng Q, Yang M. The study on the entrapment efficiency and in vitro release of puerarin submicron emulsion. AAPS PharmSciTech. 2009;10(2):376-83. doi: 10.1208/s12249-009-9216-3, PMID 19381837.

40. Chime SA, Kenechukwu FC, Attama AA. Nanoemulsionsadvances in formulation, characterization and applications in drug delivery. Appl Nanotechnol Drug Deliv. 2014. doi: $10.5772 / 58673$

41. Azeem A, Rizwan M, Ahmad FJ, Iqbal Z, Khar RK, Aqil M, Talegaonkar S. Nanoemulsion components screening and selection: A technical note. AAPS PharmSciTech.
2009;10(1):69-76. doi: 10.1208/s12249-008-9178-x, PMID 19148761.

42. Santos AC, Morais F, Simoes A, Pereira I, Sequeira JAD, Pereira Silva M, Veiga F, Ribeiro A. Nanotechnology for the development of new cosmetic formulations. Expert Opin Drug Deliv. 2019;16(4):313-30. 10.1080/17425247.2019.1585426, PMID 30793641.

43. Fytianos G, Rahdar A, Kyzas GZ. Nanomaterials in cosmetics: recent updates. Nanomaterials (Basel). 2020;10(5):1-16. doi: 10.3390/nano10050979, PMID 32443655.

44. Khan I, Saeed K, Khan I. Nanoparticles: properties, applications and toxicities. Arab J Chem. 2019;12(7):908-31. doi: 10.1016/j.arabjc.2017.05.011.

45. Yupapin P, Suwandee P. Nano-particles for cosmetic use: particle sizing, cytotoxicity test, and facial gesture monitoring model. J Cosmo Trichol. 2016;2(2):2-5. doi: 10.4172/24719323.1000112.

46. Garcia-Bilbao A, Gomez Fernandez P, Larush L, Soroka Y, Suarez Merino B, Frusic Zlotkin M, Magdassi S, Goni-de-Cerio F. Preparation, characterization, and biological evaluation of retinyl palmitate and dead sea water loaded nanoemulsions toward topical treatment of skin diseases. J Bioact Compat Polym. 2020;35(1):24-38. doi: 10.1177/0883911519885970.

47. Bernardi DS, Pereira TA, Maciel NR, Bortoloto J, Viera GS, Oliveira GC, Rocha-Filho PA. Formation and stability of oil-inwater nanoemulsions containing rice bran oil: in vitro and in vivo assessments. J Nanobiotechnology. 2011;9:44. doi: 10.1186/1477-3155-9-44, PMID 21952107.

48. Fitria. J Chem Inf Model. 2013;53(9):1689-99.

49. Bangun H, Tandiono S, Arianto A. Preparation and evaluation of chitosan-tripolyphosphate nanoparticles suspension as an antibacterial agent. J Appl Pharm Sci. 2018;8(12):147-56. doi: 10.7324/JAPS.2018.81217.

50. Ajazzuddin M, Jeswani G, Jha AK. Nanocosmetics: past, present and future trends. PNM. 2015;5(1):3-11. doi: $10.2174 / 1877912305666150417232826$.

51. Zanusso Junior G, Melo JO, Romero AL, Dantas JA, Caparroz Assef SM, Bersani Amado CA, Cuman RKN. Avaliacao da atividade antiinflamatoria do coentro (Coriandrum sativum L.) em roedores. Rev Bras Plantas Med. 2011;13(1):17-23. doi: 10.1590/S1516-05722011000100003. 